Research Article

\title{
"Vitamin D Deficiency Is More Common in Women with Autoimmune Thyroiditis: A Retrospective Study"
}

\author{
Nino Turashvili $\mathbb{D}^{1},{ }^{1}$ Lali Javashvili, ${ }^{2}$ and Elene Giorgadze ${ }^{1,3}$ \\ ${ }^{1}$ Ivane Javakhishvili Tbilisi State University, Tbilisi, Georgia \\ ${ }^{2}$ Cortex Clinic Ltd., Tbilisi, Georgia \\ ${ }^{3}$ National Institute of Endocrinology, Tbilisi, Georgia \\ Correspondence should be addressed to Nino Turashvili; ninoturashvili88@yahoo.com
}

Received 7 June 2021; Revised 19 July 2021; Accepted 11 August 2021; Published 18 August 2021

Academic Editor: Andrea Palermo

Copyright (c) 2021 Nino Turashvili et al. This is an open access article distributed under the Creative Commons Attribution License, which permits unrestricted use, distribution, and reproduction in any medium, provided the original work is properly cited.

\begin{abstract}
Background. Vitamin D is a hormone that is mainly produced in the skin upon ultraviolet B radiation exposure and has important influence on various organs. In recent years, data have been collected that vitamin D deficiency plays an important role in the development of various nonskeletal diseases, including autoimmune diseases. Chronic autoimmune thyroiditis (Hashimoto's thyroiditis) is one of the most common organ-specific autoimmune endocrine diseases. It is characterized by increased level of antithyroid peroxidase and/or antithyroglobulin antibodies in blood, which often leads to thyroid dysfunction and structural changes of the gland. There is an opinion that vitamin D deficiency may be considered as an important risk factor for development of chronic autoimmune thyroiditis, but data of various small studies are controversial. Despite the fact that Georgia is a sunny country, vitamin D deficiency is a widespread problem here. Thyroid diseases, including the chronic autoimmune thyroiditis, are also very common in Georgia. The aim of our research was to compare the level of vitamin $\mathrm{D}$ between the patients with chronic autoimmune thyroiditis and the healthy subjects. Methods. This retrospective study enrolled subjects, who were 18-70 years old and visited the clinics "Cortex" and "National Institute of Endocrinology" in 2018 or in 2019 from mid-spring to mid-summer. Data of thyroid-stimulating hormone, free thyroxine, antithyroid peroxidase antibodies, antithyroglobulin antibodies, thyroid ultrasonography, and $25(\mathrm{OH})$ vitamin D were retrospectively analysed based on medical history. In total, data of 1295 patients were collected. The statistical processing of data was performed through the SPSS 20 program. Results. The negative association between thyroid-stimulating hormone, antithyroid peroxidase antibodies, antithyroglobulin antibodies, heterogeneous parenchyma of thyroid gland, and vitamin D was found in women. Statistically significant association was not detected in men. Conclusions. Serum vitamin D is lower in women with autoimmune thyroiditis and primary hypothyroidism. Further studies are needed to evaluate the influence of vitamin D supplementation on thyroid autoantibody positivity or primary hypothyroidism.
\end{abstract}

\section{Introduction}

Vitamin $\mathrm{D}$ is a secosteroid hormone that is mainly produced in the skin upon ultraviolet B (U.V.B) radiation exposure. Other source of vitamin $\mathrm{D}$ is diet (in the form of $\mathrm{D}_{2}$ from plant sources and in the form of vitamin $\mathrm{D}_{3}$ from animal sources) and nutritional supplements (in the form of $\left.D_{3}\right)$ or ergocalciferol $\left(D_{2}\right)$. After ingestion or synthesis, vitamin $\mathrm{D}$ is hydroxylated in the liver to form 25hydroxyvitamin $\mathrm{D}\left(25(\mathrm{OH}) \mathrm{D}_{2}\right.$ or $\left.25(\mathrm{OH}) \mathrm{D}_{3}\right)$, its major circulating form, which has little biological activity.
$25(\mathrm{OH}) \mathrm{D}$ is converted in the kidney by $25(\mathrm{OH}) \mathrm{D}-1 \alpha$-hydroxylase (CYP27B1) to its bioactive hormonal metabolite 1,25 dihydroxy-vitamin $\mathrm{D}\left(1,25(\mathrm{OH})_{2} \mathrm{D}\right.$ or calcitriol) [1]. $1,25(\mathrm{OH})_{2} \mathrm{D}$ mediates its effects by binding to the vitamin $\mathrm{D}$ receptor (VDR). These receptors belong to the nuclear receptor superfamily and are expressed in nearly all tissues of the body. Therefore, vitamin D has important influence on almost all organs and cells. The concentration of serum $25(\mathrm{OH}) \mathrm{D}$ in the body is higher than serum $1,25(\mathrm{OH})_{2} \mathrm{D}$. Therefore, the serum level of $25(\mathrm{OH}) \mathrm{D}$ is typically selected to reflect the state of vitamin $\mathrm{D}$ [2]. 
The primary action of $1,25(\mathrm{OH})_{2} \mathrm{D}$ is to enhance intestinal calcium absorption and promote osteoclast function, thereby maintaining calcium and phosphorus homeostasis and bone health [3-5]. After discovery of VDR on almost all tissues, new data have emerged providing important insights into the pleiotropic effects of vitamin $\mathrm{D}$ and its potential role in a variety of extraskeletal tissues, including many that affect endocrine disease (diabetes mellitus, PCOS, Addison's disease, and autoimmune thyroid disease) [6]. Moreover, recent studies indicate that $1,25(\mathrm{OH})_{2} \mathrm{D}$ is a modulator of both the innate and adaptive immune system. Immune cells such as monocytes, macrophages, dendritic cells, T-lymphocytes, and B-lymphocytes are targets for active vitamin D [7]. Active vitamin D regulates T cells: It inhibits the proliferation of T helper 1 (Th1) and decreases production of cytokines such as interleukin 2 (IL-2), tumor necrosis factor, and interferon $\gamma($ IFN- $\gamma)$. Vitamin $\mathrm{D}$ shifts the polarization of T cells from Th1 toward Th2 that produce IL-4 and IL-5. A third group of Th cells known to be influenced by vitamin $\mathrm{D}$ are IL-17-secreting T cells (Th17 cells). 1,25(OH) ${ }_{2} \mathrm{D}$ inhibits IL-17 production. Vitamin D promotes apoptosis of dendritic cell (DC) and inhibits its differentiation and maturation. Expression of major histocompatibility complex (MHC) II on DC is downregulated by vitamin D. Tolerogenic DCs arrest the development of autoimmune diseases [8].

Hashimoto's thyroiditis (HT) is a chronic autoimmune thyroiditis, which is one of the most common organ-specific autoimmune disorder. It is characterized by increased levels of antithyroid peroxidase (anti-TPO) and/or antithyroglobulin (anti-TG) antibodies in serum, which often leads to thyroid dysfunction [9].

HT patients have a high level of Th1 cells, which produce the cytokine IFN- $\gamma$. Recent studies indicated that secretion of cytokines from Th17 is also involved in the development of autoimmune thyroid disease (AITD). IFN- $\gamma$ and IFN-17A mRNA expression is significantly higher in HT patients than in healthy controls $[10,11]$.

According to some studies, $1,25(\mathrm{OH})_{2} \mathrm{D}$ may suppress the autoimmune process in HT at several levels, including suppression of dendritic cell-dependent T-cell activation, and decrease proliferation of Th1 cells and the synthesis of Th 1 cell cytokines such as IFN-y. Vitamin D may also inhibit the expression of MHCII surface HLA-DR antigens on thyrocytes by inhibiting the synthesis of IFN-y. In this way, vitamin $\mathrm{D}$ might decrease autoantibodies that react with thyroid antigens $[8,12,13]$.

Recent studies have suggested a possible role of vitamin $\mathrm{D}$ in increasing the risk of HT development [14]; however, data regarding the possible role of vitamin $\mathrm{D}$ in the pathogenesis of HT are controversial: some studies suggest that vitamin D deficiency may be related to pathogenesis of HT and its supplementation could be beneficial for the treatment of HT $[8,10,15-25]$. Other studies indicate that HT is not associated with vitamin $\mathrm{D}$ deficiency relative to a control group [26-30].

Despite the fact that Georgia is a sunny country, vitamin $\mathrm{D}$ deficiency or its insufficiency is a widespread problem here. Thyroid diseases, including the chronic autoimmune thyroiditis, are also very common in Georgia.
The aim of our research was to compare the level of vitamin $\mathrm{D}$ between the patients with chronic autoimmune thyroiditis and the healthy subjects. The primary objective of this study was to determine the association between thyroidstimulating hormone (TSH), free thyroxine (FT4), antiTPO, anti-TG, and vitamin D, as well as structural changes in the thyroid gland and vitamin D. Research on this issue has never been conducted in Georgia. Research hypothesis was the following: Patients with chronic autoimmune thyroiditis may have lower level of vitamin $\mathrm{D}$, compared to healthy subjects.

\section{Methods}

This retrospective study enrolled subjects, who were 18-70 years old and visited the clinics "Cortex" and "National Institute of Endocrinology" in 2018 or in 2019 from midspring to mid-summer. The study protocol was approved by the medical ethics committees of these clinics.

Retrospective selection of the study population was performed based on the patient's history: age 18-70 years and subjects who had lived in Georgia for a year or longer. Exclusion criteria: patients with metabolic bone diseases, primary hyperparathyroidism, hypoparathyroidism, central hypothyroidism, history of thyroidectomy, renal disorders, liver disorders, malabsorption syndromes, type 1 diabetes, obesity, granuloma-forming disorders, or epilepsy treated by anticonvulsants or patients with malignancy, immunodeficiency, and those on chronic medications that could interfere with thyroid hormone or vitamin D metabolism. Pregnant or lactating women and those who had received levothyroxine, vitamin $\mathrm{D}$, or calcium supplementation in the last 6 months were also excluded.

In total, data of 1295 patients were collected. All patients had vitamin D and TSH measurement. From total 1295 participants, 866 subjects had FT4 data, 1263 had anti-TPO data, 295 had anti-TG data, and 262 had both anti-TPO and anti-TG data. Ultrasonographically, 262 subjects had the information about thyroid gland volume and echogenicity. In total, 85 subjects had thyroid nodules and information about nodule size and characteristics.

The association between study characteristics and vitamin D was statistically analysed. The laboratory and ultrasound examinations of each patient were performed within one week. The chemiluminescence immunoassay was used to analyze TSH, FT4, anti-TPO, and anti-TG levels, and the fluorescent enzyme immunoassay was used to measure vitamin D level. The thyroid and vitamin D tests have been performed on the "Tosoh AIA-900" in the same laboratory.

Serum $25(\mathrm{OH}) \mathrm{D}$ level of $\geq 30 \mathrm{ng} / \mathrm{ml}$ was considered as normal level and 20 to $29 \mathrm{ng} / \mathrm{ml}$ as vitamin D insufficiency, whereas serum $25(\mathrm{OH}) \mathrm{D}$ levels of $<20 \mathrm{ng} / \mathrm{ml}$ were considered as an indicative of vitamin D deficiency [31]. Serum anti-TPO $>34 \mathrm{IU} / \mathrm{mL}$ and/or anti-TG $>115 \mathrm{IU} / \mathrm{mL}$ were considered as autoantibody positivity. Normal range for TSH was $0.27-4.2 \mu \mathrm{IU} / \mathrm{ml}$. Normal level of FT4 was $0.93-1.7 \mathrm{ng} / \mathrm{dl}$ (according to the local laboratory reference range). The total volume of thyroid $>18 \mathrm{~cm}^{3}$ in female and $>25 \mathrm{~cm}^{3}$ in male indicate thyroid enlargement [32]. Elevated 
TSH value was used to declare primary hypothyroidism. Elevated anti-TPO and/or anti-TG levels were used to establish the diagnosis of autoimmune thyroiditis (Hashimoto's thyroiditis).

For statistical analysis, the study population was divided according to gender and age groups for women only $(<45$ years and $>45 \mathrm{y}$.).

The statistical processing of data was performed through the Statistical Package for the Social Sciences (SPSS) version 20 program. After the data grouping, their percentages were comparable to vitamin D subgroups (up to 20- deficiency, 20-29- insufficiency, and 30 and higher- normal), whose credibility was estimated by the $\chi^{2}$ test (chi-square test).

Descriptive analysis was conducted for the same groups of vitamin $\mathrm{D}$, whose credibility was assessed by the ANOVA test. A $p$ value of $<0.05$ was considered as statistically significant.

\section{Results}

The data of 1295 patients were collected, including 1097 women (84.71\%) and 198 men (15.29\%). The age distribution of women patients was as following: $849(77.39 \%)$ were $<45$ years old and $248(22.61 \%)>45$ years old.

TSH and 25(OH) vitamin D was performed in all study participants. Elevated TSH level was observed in 141 subjects (10.89\%), while the majority, 1154 (89.11\%), had normal TSH level. Mean vitamin D value was low in both TSH level groups and corresponded to vitamin D deficiency category; the lower vitamin D level was observed in patients with primary hypothyroidism. Our study revealed a negative association between TSH and vitamin D levels in the total study population $(p=0.008)$.

When we analysed the study population according to gender, we observed that 121 female subjects (11.03\%) had high TSH levels. Vitamin D levels were generally low in this subgroup, and only $5.5 \%$ patients with euthyroidism and $1.7 \%$ with primary hypothyroidism had normal vitamin D status. According to our study results, a statistically significant negative association was observed between TSH and vitamin D levels in the total women population $(p=0.01)$ and those whose age was $<45$ years $(p=0.036)$. The association between TSH level and vitamin D was not statistically significant in women $>45$ years of age $(p=0.232)$ and in male study participants $(p=0.465)$.

The association between FT4 and vitamin D was not detected neither in the total study population nor in groups divided according to gender or age.

The negative association between anti-TPO and vitamin D was statistically significant in the whole study group $(p=0.011)$ as well as in the group of women $(p=0.021)$ and women $<45$ years of age $(p=0.044)$.

The association between anti-TG and vitamin D was only observed in the group of women $(p=0.022)$.

The association between both antibodies and vitamin $\mathrm{D}$ was not detected in neither group.

Thyroid ultrasonography data of 262 patients (210 females and 52 males) were analysed.
A statistically significant negative association between heterogeneous parenchyma of thyroid and vitamin $\mathrm{D}$ was revealed in women $(p=0.048)$.

There was not also any association between total thyroid volume and vitamin $\mathrm{D}$ in women, as well as between the number of nodules and vitamin $\mathrm{D}$ and between the size of the largest nodule and vitamin D.

There was not also any association between total thyroid volume and vitamin $\mathrm{D}$, heterogeneous parenchyma and vitamin $\mathrm{D}$, the number of nodules and vitamin $\mathrm{D}$, and between the size of largest nodule and vitamin $\mathrm{D}$ in the whole group as well as in men.

\section{Discussion}

Our study demonstrated the significant negative association between TSH and vitamin D, between anti-TPO and vitamin $\mathrm{D}$, and anti-TG and vitamin $\mathrm{D}$ in women, as well as between the heterogeneous parenchyma of thyroid and vitamin D level in women.

As far as we know, this is the first research in this field in Georgia.

From total 1295 patients, $70.6 \%$ had vitamin D deficiency, $24.1 \%$ had vitamin D insufficiency, and only 5.3\% had normal vitamin $\mathrm{D}$ level. This is an unexpected result because Georgia is a sunny country. Vitamin D deficiency was higher in hypothyroidism patients $(78.7 \%$ compared to $69.6 \%$ in euthyroid people), and normal vitamin D level also was less common in hypothyroidism patients $(2.8 \%$ vs. $5.6 \%)$. This result confirms our hypothesis that there is a connection between high TSH level and vitamin D deficiency.

We collected data of 1263 patients about anti-TPO and vitamin D. According to them, $71.2 \%$ had vitamin D deficiency, $23.7 \%$ had vitamin D insufficiency, and only $5.1 \%$ had normal vitamin D levels. Vitamin D deficiency was higher in patients with elevated anti-TPO (74.9\% vs. $69.7 \%)$. Between 295 patients with data of anti-TG and vitamin D, 101 subjects (34.24\%) had high anti-TG. Vitamin D deficiency was higher in this group, but not statistically significant.

From 866 data of FT4 and vitamin D, only 5.1\% had low FT4. Vitamin D deficiency was also more common in this group (79.5 vs. $68.7 \%$ ), but not statistically significant.

According to this result, we think that vitamin D deficiency is a widespread problem, but it is more common in patients in autoimmune thyroiditis and primary hypothyroidism. We think that this can be explained by vitamin D's immunomodulatory ability: Vitamin $\mathrm{D}$ deficiency may promote the production of various cytokines, which causes the development of autoimmune thyroiditis and often leads to decreased thyroid function.

According to gender analysis, we noted that $11.03 \%$ of women and $10.1 \%$ of men had primary hypothyroidism, but vitamin $\mathrm{D}$ deficiency was statistically significant only in the female group. Vitamin D was low in both groups, but the mean of vitamin D was 16.8573 in female and 18.2870 in the male group. This indicates that the link between primary 
hypothyroidism and vitamin D deficiency was found only in female, not in male.

Anti-TPO had been measured in 1073 female and 190 male. $30.75 \%$ in women and $13.16 \%$ in men had high antiTPO, as well as $37.45 \%$ of 243 female and $19.23 \%$ of 52 male had high anti-TG level. A statistically significant negative association was detected only in women. This result can be explained by the fact that Hashimoto's thyroiditis is more common in women and vitamin $\mathrm{D}$ deficiency has been found more in women, than in men; therefore, there is a possible link between autoimmune thyroiditis and vitamin $\mathrm{D}$ deficiency, especially in female.

In our study, we divided the female group by age: $77.39 \%$ $<45$ years of age and $22.61 \%>45$ years of age. We found a significant negative association between TSH, anti-TPO, and vitamin $\mathrm{D}$ only in premenopausal women. We suggest a possible link between vitamin $\mathrm{D}$ and estrogen in the development of AITD.

So, our retrospective study indicates that vitamin $\mathrm{D}$ deficiency is more common in patients (especially in women up to 45 years of age) with autoimmune thyroiditis and primary hypothyroidism than in the healthy group.

However, there are many studies with different results. For example, Yasmeh et al. studied whether vitamin D deficiency was associated with HT. Different from our study, they concluded that HT was not associated with higher rates of vitamin D deficiency relative to a control group neither in women nor in men [26]. But, in contrast to our data, in their study, the mean $25(\mathrm{OH}) \mathrm{D}$ levels for the HT and control groups were 30.75 vs. $27.56 \mathrm{ng} / \mathrm{mL}$ in females. So, none of the females were vitamin $\mathrm{D}$ deficient.

Similar to our research, Choi et al. described in their study that the mean of serum $25(\mathrm{OH}) \mathrm{D}$ levels was significantly lower in the TPO-Ab(+) female subjects, especially in premenopausal women. They also suggested a possible crosstalk between vitamin $\mathrm{D}$ and estrogen in the development of AITD [33]. Wang et al. revealed that low serum $25(\mathrm{OH}) \mathrm{D}$ is related to the presence of TG-Ab in females [19]. In their study, the prevalence of vitamin $\mathrm{D}$ deficiency (78.3\%) and insufficiency (20\%) was higher in TG-Abpositive subjects and women had higher serum anti-TG levels and lower serum $25(\mathrm{OH}) \mathrm{D}_{3}$ than men. The authors of the survey also think about a possible crosstalk between vitamin D and X chromosome in the development of AITDs. Kim believes that serum 25(OH)D level was significantly negatively correlated with serum TSH level [18], but Musa et al. did not report any association between $25(\mathrm{OH})$ vitamin $\mathrm{D}_{3}$ level and hypothyroidism among females [30]. We think that the reason of this is the difference between our studies. There was a fewer number of participants (fifty-eight participants in each group of the study). Du et al. found that the incidence of thyroid nodules is lower in high level of serum $25(\mathrm{OH}) \mathrm{D}_{3}$ and serum $25(\mathrm{OH}) \mathrm{D}_{3}$ may be a protective factor for thyroid nodules [2]. However, we did not find any connection between vitamin $\mathrm{D}$ and thyroid nodules. The reason for this might be the high number of patients with thyroid nodules in their study. No significant association between serum $25(\mathrm{OH}) \mathrm{D}_{3}$ and the size of the largest nodule was revealed in our study. In our study, we found the negative association between heterogeneous thyroid parenchyma and low vitamin D. Heterogeneity of parenchyma is a characteristic sign for chronic autoimmune thyroiditis: lymphocytic infiltration of the thyroid leads to gradual destruction and fibrous replacement of the thyroid parenchymal tissue. In addition, Nalbant et al. showed that parenchymal blood supply of thyroid gland decreased and microvascular resistance increased in vitamin D insufficiency/deficiency. Therefore, vitamin D insufficiency/deficiency might lead to severe parenchymal injury in HT patients [34].

It should be noted that nowadays, there is a lack of consensus surrounding what constitutes optimal serum concentrations of vitamin D. Adequate serum 25-hydroxyvitamin D concentrations have been suggested to be $20 \mathrm{ng} /$ $\mathrm{ml}(50 \mathrm{nmol} / \mathrm{l})$ [35] or $30 \mathrm{ng} / \mathrm{ml}(75 \mathrm{nmol} / \mathrm{l})$ [31]. However, at present, there is no agreement on "normal levels" of $25(\mathrm{OH}) \mathrm{D}$. Several methods have been used in the quantification of vitamin D. Serum total $25(\mathrm{OH}) \mathrm{D}$ concentration remains the critical measurement for defining vitamin $D$ status. Serum total $25(\mathrm{OH}) \mathrm{D}$ is defined as the sum of the concentrations of $25(\mathrm{OH}) \mathrm{D}_{3}$ and $25(\mathrm{OH}) \mathrm{D}_{2}$. Measurement of vitamin D status, based on currently available data, should not include the concentration of 3 -epi- $25(\mathrm{OH}) \mathrm{D}_{3}$ or any other vitamin $\mathrm{D}$ metabolites. The analytical difficulties related to the measurement of vitamin D metabolites may lead to misclassification of 'low' vitamin D status. The variations in analytical techniques and assays may cause the large variability in laboratory measurements of vitamin D status and the systematic "errors" [36-45]. In our study, the serum $25(\mathrm{OH}) \mathrm{D}$ level of $\geq 30 \mathrm{ng} / \mathrm{ml}$ was considered as the normal level and 20 to $29 \mathrm{ng} / \mathrm{ml}$ as vitamin D insufficiency, whereas serum $25(\mathrm{OH}) \mathrm{D}$ levels of $<20 \mathrm{ng} / \mathrm{ml}$ were considered as an indicative of vitamin D deficiency. From total 1295 patients, $70.6 \%$ had vitamin D level $<20 \mathrm{ng} / \mathrm{ml}$. So, we think that despite these different cutoff levels, the association between hypovitaminosis D and autoimmune thyroiditis would probably remain the same, even if we were to use a different level of vitamin D normality $(\geq 20 \mathrm{ng} / \mathrm{ml})$, but this is a subject of another study. We considered seasonal influences on vitamin D status [46], and therefore, for our retrospective study, we collected patients' data whose thyroid and vitamin D laboratory tests and thyroid ultrasound examinations were performed from mid-spring to mid-summer in 2018 or in 2019 (within one week for each patient). However, there are some limitations in our study: We could not analyse data according to patients' outdoor activity, BMI, and smoking status. Due to the analytical difficulties, in our retrospective study, we have no data about all metabolites levels of vitamin D. The number of men patients was also limited.

Thus, the results of various studies are contradictory, but for the first time, our research revealed the wide distribution of vitamin D deficiency in the population of Georgia and the possible link between the chronic autoimmune thyroiditis and primary hypothyroidism and vitamin $\mathrm{D}$ deficiency. However, up to now, we do not have the evidence-based data showing the efficacy of the therapeutic effect of vitamin $\mathrm{D}$ in improving primary hypothyroidism and decreasing thyroid autoantibody level. 


\section{Conclusions}

In summary, serum vitamin $\mathrm{D}$ is lower in women with autoimmune thyroiditis and primary hypothyroidism. Further studies are needed to evaluate the influence of vitamin D supplementation on thyroid autoantibody positivity or primary hypothyroidism.

\section{Abbreviations}

$\begin{array}{ll}\text { Th: } & \text { T helper } \\ \text { IL: } & \text { Interleukin } \\ \text { IFN: } & \text { Interferon } \\ \text { DC: } & \text { Dendritic cell } \\ \text { HT: } & \text { Hashimoto's thyroiditis } \\ \text { Anti-TPO: } & \text { Antithyroid peroxidase antibodies } \\ \text { Anti-TG: } & \text { Antithyroglobulin antibodies } \\ \text { AITD: } & \text { Autoimmune thyroid disease } \\ \text { TSH: } & \text { Thyroid-stimulating hormone } \\ \text { FT4: } & \text { Free thyroxine. }\end{array}$

\section{Data Availability}

All data generated or analysed during this study are included in this published article.

\section{Ethical Approval}

The study protocol was approved by the medical ethics committees of the clinics "Cortex" and "National Institute of Endocrinology."

\section{Consent}

No consent was required for this study.

\section{Conflicts of Interest}

The authors declare no conflicts of interest.

\section{Authors' Contributions}

All authors read and approved the final manuscript.

\section{Supplementary Materials}

Table 1: association between TSH and vitamin D. Table 2: association between anti-TPO and vitamin D. Table 3: association between anti-TG and vitamin $\mathrm{D}$ in female. Table 4: association between heterogeneous parenchyma of thyroid and vitamin D in female. (Supplementary Materials)

\section{References}

[1] J. Jameson, Harrison's Endocrinology, 3E, p. 396, McGraw-Hill Education, NY, USA, 2013.

[2] X. Du, Y. Liu, C. Zhao, J. Fang, X. Wang, and L. Wei, "Changes of serum $25(\mathrm{OH}) \mathrm{D}_{3}$ and IGF-1 levels in patients with thyroid nodules," BMC Endocrine Disorders, vol. 19, no. 1, p. 48, 2019.
[3] R. Cesareo, M. Iozzino, L 'D'onofrio et al., "Effectiveness and safety of calcium and vitamin D treatment for postmenopausal osteoporosis," Minerva Endocrinologica, vol. 40, no. 3, pp. 231-237, 2015.

[4] C. Liu, X. Kuang, K. Li, X. Guo, Q. Deng, and D. Li, "Effects of combined calcium and vitamin $\mathrm{D}$ supplementation on osteoporosis in postmenopausal women: a systematic review and meta-analysis of randomized controlled trials," Food \& Function, vol. 11, no. 12, pp. 10817-10827, 2020.

[5] T. Yoshida and P. H. Stern, "How vitamin D works on bone," Endocrinology and Metabolism Clinics of North America, vol. 41, no. 3, pp. 557-569, 2012.

[6] A. J. Thompson, S. E. Baranzini, J. Geurts, B. Hemmer, and O. Ciccarelli, "Multiple sclerosis," The Lancet, vol. 391, no. 10130, pp. 1622-1636, 2018.

[7] W. Ke, T. Sun, Y. Zhang et al., "25-Hydroxyvitamin D serum level in Hashimoto's thyroiditis, but not Graves' disease is relatively deficient," Endocrine Journal, vol. 64, no. 6, pp. 581-587, 2017.

[8] A. D. Unal, O. Tarcin, H. Parildar, O. Cigerli, H. Eroglu, and N. G. Demirag, "Clinical immunology vitamin D deficiency is related to thyroid antibodies in autoimmune thyroiditis," Central European Journal of Immunology, vol. 39, no. 4, pp. 493-497, 2014.

[9] A. McGrogan, H. E. Seaman, J. W. Wright, and C. S. De Vries, "The incidence of autoimmune thyroid disease: a systematic review of the literature," Clinical Endocrinology, vol. 69, no. 5, pp. 687-696, 2008.

[10] J. Wang, S. Lv, G. Chen et al., "Meta-analysis of the association between vitamin $\mathrm{D}$ and autoimmune thyroid disease," $\mathrm{Nu}$ trients, vol. 7, no. 4, pp. 2485-2498, 2015.

[11] D. Li, W. Cai, R. Gu et al., "Th17 cell plays a role in the pathogenesis of Hashimoto's thyroiditis in patients," Clinical Immunology, vol. 149, no. 3, pp. 411-420, 2013.

[12] M. T. Cantorna, Y. Zhu, M. Froicu, and A. Wittke, "Vitamin D status, 1,25-dihydroxyvitamin $\mathrm{D}_{3}$, and the immune system," American Journal of Clinical Nutrition, vol. 80, no. 6, pp. 1717S-1720S, 2004.

[13] S. Kivity, N. Agmon-Levin, M. Zisappl et al., "Vitamin D and autoimmune thyroid diseases," Cellular \& Molecular Immunology, vol. 8, no. 3, pp. 243-247, 2011.

[14] K. Vondra, L. Stárka, and R. Hampl, "Vitamin D and thyroid diseases," Physiological Research, vol. 64, no. Suppl 2, pp. S95-S100, 2015.

[15] M. Nodehi, A. Ajami, M. Izad et al., "Effects of vitamin D supplements on frequency of CD4+ T-cell subsets in women with Hashimoto's thyroiditis: a double-blind placebo-controlled study," European Journal of Clinical Nutrition, vol. 73, no. 9, pp. 1236-1243, 2019.

[16] S. Wang, Y. Wu, Z. Zuo, Y. Zhao, and K. Wang, "The effect of vitamin D supplementation on thyroid autoantibody levels in the treatment of autoimmune thyroiditis: a systematic review and a meta-analysis," Endocrine, vol. 59, no. 3, pp. 499-505, 2018.

[17] E. E. Mazokopakis, M. G. Papadomanolaki, K. C. Tsekouras, A. D. Evangelopoulos, D. A. Kotsiris, and A. A. Tzortzinis, "Is vitamin $\mathrm{D}$ related to pathogenesis and treatment of Hashimoto's thyroiditis?" Hellenic Journal of Nuclear Medicine, vol. 18, no. 3, pp. 222-227, 2015.

[18] D. Kim, "Low vitamin D status is associated with hypothyroid Hashimoto's thyroiditis," Hormones (Athens, Greece), vol. 15, no. 3, pp. 385-393, 2016.

[19] X. Wang, J. Zynat, Y Guo et al., "Low serum vitamin D is associated with anti-thyroid-globulin antibody in female 
individuals," International journal of endocrinology, vol. 2015, Article ID 285290, 2015.

[20] G. Muscogiuri, D. Mari, S. Prolo et al., "25 hydroxyvitamin D deficiency and its relationship to autoimmune thyroid disease in the elderly," International Journal of Environmental Research and Public Health, vol. 13, no. 9, p. 850, 2016.

[21] D. Kim, "The role of vitamin D in thyroid diseases," International Journal of Molecular Sciences, vol. 18, no. 9, p. 1949, 2017.

[22] S. Chaudhary, D. Dutta, M. Kumar et al., "Vitamin D supplementation reduces thyroid peroxidase antibody levels in patients with autoimmune thyroid disease: an open-labeled randomized controlled trial," Indian journal of endocrinology and metabolism, vol. 20, no. 3, pp. 391-398, 2016.

[23] A. Talaei, F. Ghorbani, and Z. Asemi, "The effects of vitamin D supplementation on thyroid function in hypothyroid patients: a randomized, double-blind, placebo-controlled trial," Indian Journal of Endocrinology and Metabolism, vol. 22, no. 5, p. 584, 2018.

[24] J. Ma, D. Wu, C. Li et al., "Lower serum 25-hydroxyvitamin D level is associated with 3 types of autoimmune thyroid diseases," Medicine, vol. 94, no. 39, Article ID e1639, 2015.

[25] M. S. Arslan, O. Topaloglu, B. Ucan et al., "Isolated vitamin D deficiency is not associated with nonthyroidal illness syndrome, but with thyroid autoimmunity," The Scientific World Journal, vol. 2015, Article ID 239815, 2015.

[26] J. Yasmeh, F. Farpour, V. Rizzo, S. Kheradnam, and I. Sachmechi, "Hashimoto thyroiditis not associated with vitamin D deficiency," Endocrine Practice, vol. 22, no. 7, pp. 809-813, 2016.

[27] P. V. Anaraki, A. Aminorroaya, M. Amini, A. Feizi, B. Iraj, and A. Tabatabaei, "Effects of vitamin D deficiency treatment on metabolic markers in Hashimoto thyroiditis patients," Journal of Research in Medical Sciences, vol. 21, no. 1, 2016.

[28] P. V. Anaraki, A. Aminorroaya, M. Amini et al., "Effect of vitamin $\mathrm{D}$ deficiency treatment on thyroid function and autoimmunity markers in Hashimoto's thyroiditis: a doubleblind randomized placebo-controlled clinical trial," Journal of Research in Medical Sciences, vol. 22, no. 1, 2017.

[29] K. V. Knutsen, A. A. Madar, M. Brekke et al., "Effect of vitamin D on thyroid autoimmunity: a randomized, doubleblind, controlled trial among ethnic minorities," Journal of the Endocrine Society, vol. 1, no. 5, pp. 470-479, 2017.

[30] I. R. Musa, G. I. Gasim, S. Khan, I. A. Ibrahim, H. Abo-alazm, and I. Adam, "No association between $25(\mathrm{OH})$ vitamin D level and hypothyroidism among females," Open Access Macedonian Journal of Medical Sciences, vol. 5, no. 2, pp. 126-130, 2017.

[31] M. F. Holick, N. C. Binkley, H. A. Bischoff-Ferrari et al., "Evaluation, treatment, and prevention of vitamin D deficiency: an Endocrine Society clinical practice guideline," The Journal of Clinical Endocrinology \& Metabolism, vol. 96, no. 7, pp. 1911-1930, 2011.

[32] I. I. Dedov, G. A. Melnichenko, and V. V. Fadeev, Endocrinology: The Textbook for Universities, GEOTAR-Media, Russia, 2007.

[33] Y. M. Choi, W. G. Kim, T. Y. Kim et al., "Low levels of serum vitamin $\mathrm{D}_{3}$ are associated with autoimmune thyroid disease in pre-menopausal women," Thyroid, vol. 24, no. 4, pp. 655-661, 2014.

[34] A. Nalbant, A. Aydin, A. Karacan, A. Onmez, A. Tamer, and H. Cinemre, "Association of vitamin D insufficiency/deficiency with thyroid artery Doppler ultrasonography in patients with Hashimoto thyroiditis," Pakistan Journal of Medical Sciences, vol. 33, no. 2, pp. 295-299, 2017.

[35] A. C. Ross, C. L. Taylor, A. L. Yaktine, and H. B. Del Valle, "Dietary reference intakes for adequacy: calcium and vitamin D. InDietary reference intakes for calcium and vitamin D 2011," Washington, DC, USA, 2011.

[36] C. S. Stokes, F. Lammert, and D. A. Volmer, "Analytical methods for quantification of vitamin $\mathrm{D}$ and implications for research and clinical practice," Anticancer Research, vol. 38, no. 2, pp. 1137-1144, 2018.

[37] C. T. Sempos, R. A. Durazo-Arvizu, N. Binkley, J. Jones, J. M. Merkel, and G. D. Carter, "Developing vitamin D dietary guidelines and the lack of 25-hydroxyvitamin D assay standardization: the ever-present past," The Journal of Steroid Biochemistry and Molecular Biology, vol. 164, pp. 115-119, 2016.

[38] Del Valle H. B., Yaktine A. L., Taylor C. L., Ross A. C., editors. Dietary Reference Intakes for Calcium and Vitamin D.

[39] C. T. Sempos, A. C. Heijboer, D. D. Bikle et al., "Vitamin D assays and the definition of hypovitaminosis D: results from the first international conference on controversies in vitamin D," British Journal of Clinical Pharmacology, vol. 84, no. 10, pp. 2194-2207, 2018.

[40] R. Cesareo, R. Attanasio, M. Caputo et al., "Italian association of clinical endocrinologists (AME) and Italian chapter of the American association of clinical endocrinologists (AACE) position statement: clinical management of vitamin $\mathrm{D}$ deficiency in adults," Nutrients, vol. 10, no. 5, p. 546, 2018.

[41] A. C. Ross, J. E. Manson, S. A. Abrams et al., "The 2011 report on dietary reference intakes for calcium and vitamin $\mathrm{D}$ from the Institute of Medicine: what clinicians need to know," The Journal of Clinical Endocrinology \& Metabolism, vol. 96, no. 1, pp. 53-58, 2011.

[42] N. Binkley and G. D. Carter, "Toward clarity in clinical vitamin D status assessment," Endocrinology and Metabolism Clinics of North America, vol. 46, no. 4, pp. 885-899, 2017.

[43] C. A. Nowson, J. J. McGrath, P. R. Ebeling et al., "Vitamin D and health in adults in Australia and New Zealand: a position statement," Medical Journal of Australia, vol. 196, no. 11, pp. 686-687, 2012.

[44] N. Binkley, C. T. Sempos, and Vitamin D. Standardization Program (VDSP), "Standardizing vitamin D assays: the way forward," Journal of Bone and Mineral Research, vol. 29, no. 8, pp. 1709-1714, 2014.

[45] L. N. Bjerg, J. R. Halgreen, S. H. Hansen, H. A. Morris, and N. R. Jørgensen, "An evaluation of total 25-hydroxyvitamin D assay standardization: where are we today?" The Journal of Steroid Biochemistry and Molecular Biology, vol. 190, pp. 224-233, 2019.

[46] M. Štefanić and S. Tokić, "Serum 25-hydoxyvitamin D concentrations in relation to Hashimoto's thyroiditis: a systematic review, meta-analysis and meta-regression of observational studies," European Journal of Nutrition, vol. 59, no. 3 , pp. 859-872, 2020. 